\title{
Claudication: A Teachable Moment or Missed Opportunity!
}

\section{Ayad Jindeel*}

Department of Vascular Surgery and Medicine, Essentia Health Duluth Clinic, Minnesota, USA

*Corresponding author: Ayad Jindeel, Department of Vascular Surgery and Medicine, Essentia Health Duluth Clinic, 400 East 3rd street, Duluth MN 55812, USA, E-mail: aj1med@aol.com

\begin{abstract}
Claudication is a common manifestation of Peripheral Arterial Disease (PAD) and atherosclerosis. Patients with claudication are at increased risk of cardiovascular events and limb-related complications. Optimal medical therapy is effective in relieving patient's symptoms and reducing their risk of cardiovascular events.

Case: A 54-year-old male with hypertension, hyperlipidemia, tobacco use and sedentary lifestyle presented with left lower extremity exertional pain. Lower extremity segmental arterial study was consistent with the presence of Lower extremity arterial disease. He was managed medically with resolution of his claudication symptoms and improvement of his segmental study.

Conclusion: Medical management of patients with claudication is extremely effective and should be provided to all patients with claudication, even those who undergo revascularization to reduce their risk of cardiovascular events, improve their claudication symptoms and general health.
\end{abstract}

\section{Keywords}

Claudication, Peripheral Arterial Disease (PAD)

\section{Introduction}

The prevalence of Peripheral Arterial Disease (PAD) among adults aged $>40$ years is $4.3 \%$ and $13.4 \%$ among adults aged $>65$ years. The prevalence of claudication is $1.9 \%$ in males and $0.8 \%$ in females aged $>40$ years. The majority of patients with PAD are either asymptomatic or have atypical leg symptoms [1-3]. Major risk factors for lower extremity arterial disease are increasing age, tobacco use, diabetes, hypertension and Hyperlipidemia $[1,3]$. Atherosclerosis is the most common cause of PAD, but other causes like embolism, vasculitis, Buerger's disease, fibromuscular dysplasia and popliteal entrapment syndrome should be considered.
The majority of patients with lower extremity arterial disease presents with atypical leg complaints or are asymptomatic [4]. Patients with claudication usually present with a variety of symptoms like dull aching, tightness, pain, weakness or fatigue in the calves, thighs or buttocks triggered by physical activities. Patients with claudication usually have co-morbid conditions that could cause different types of pain in the same limb like osteoarthritis, bursitis and neurological causes. The location and severity of symptoms depends on the level and degree of their arterial disease. Symptoms resolve after resting for at least few minutes. Symptoms that start or resolve immediately after initiating or stopping physical activities suggest alternative diagnosis especially musculoskeletal and neurological causes. Also, sitting is not required for resolution of claudication and symptoms that resolve only after sitting or lying down are suggestive of musculoskeletal or neurological causes. Some patients will not complain about their claudication especially the elderly patients because they attribute them to their age or other coexisting conditions such as osteoarthritis. It is important to elicit information regarding the onset of symptoms as gradual or sudden, unilateral or bilateral, and whether the symptoms were triggered by usual physical activities to the patient or after the patient started a new type of physical activity. Ischemic rest pain suggests severe peripheral arterial disease and could be present with severe claudication. It usually affects the toes and feet and is worse with recumbence and better with dangling the feet down. Patients with severe PAD may have coldness, paresthesia, weakness and atrophy of affected foot or limb.

\section{Physical examination}

Of patients with suspected peripheral arterial disease and claudication needs to be thorough and detailed.

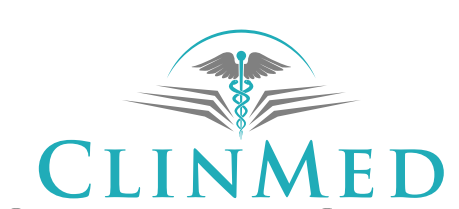

INTERNATIONAL LIBRARY

Citation: Jindeel A (2017) Claudication: A Teachable Moment or Missed Opportunity!. Clin Med Rev Case Rep 4:186. doi.org/10.23937/2378-3656/1410186

Received: July 25, 2017: Accepted: September 21, 2017: Published: September 23, 2017

Copyright: (C) 2017 Jindeel A, et al. This is an open-access article distributed under the terms of the Creative Commons Attribution License, which permits unrestricted use, distribution, and reproduction in any medium, provided the original author and source are credited. 


\section{Inspection}

Patients' ability to transfer from chair to bed or vice versa and their ability to walk even several steps should be noted. If a patient is unable to put weight on one leg, this could indicate the presence of musculoskeletal etiology such as arthritis. If a patient has difficulty transferring from lying down in bed to sitting up and transferring to chair secondary to back pain, this could suggest the presence of radiculopathy. Lower extremities should be inspected for any unilateral or bilateral deformities, atrophy, amputation, ulcer, gangrene, skin and nail dystrophic changes, discoloration and old scars. The presence of varicose veins and swelling should be documented. Socks and shoes should be inspected for discharge and proper fitting, especially in patients with foot lesions. Dependent rubor of the feet and pallor are suggestive of severe ischemia.

\section{Palpation}

Of the legs and feet for warmth, coldness, tenderness, and peripheral pulses, is essential. Cold feet could indicate ischemia and warmth could suggest infection. Normal pedal pulses reduce the possibility of significant lower extremity arterial disease. Dorsalis pedis is congenitally absent in some people. Diminished femoral pulse is caused by aortoiliac lesions. The presence of normal femoral pulse with diminished popliteal artery pulse indicates mostly superficial femoral artery pathology. Irregular pulse could indicate cardiac arrhythmias and possible cardiac source of embolism as a cause of PAD. Sensation should be tested, especially in those with diabetes or in those with symptoms like numbness. Patients with peripheral neuropathy could have severe ischemia with minimal symptoms (rest pain equivalent). Palpation of the aorta and peripheral pulsation from the radial, carotid, femoral, popliteal, dorsalis pedis and posterior tibial arteries is essential to detect irregular rhythm, absence (0), diminished (1+) or normal $(2+)$ pulsation and the presence of aneurysm.

If pedal pulses are not palpable or diminished, a hand held Doppler, if available, could be used to localize and investigate pedal pulses further. The location of the DP pulse is lateral to the extensor pollicis longus tendon along the dorsum of mid foot and the posterior tibial pulse is posterior to medial malleolus. Absent doppler signals suggest congenitally absent pulse or occlusion of that artery. Doppler signal is characterized as triphasic, biphasic or monophasic. Monophasic signal suggest the presence of significant arterial disease or inflammation.

\section{Auscultation}

For abnormal heart sounds and bruit over the carotid, renal and femoral arteries. The presence of arterial bruit is a specific sign for significant arterial disease.

\section{Differential Diagnosis}

The most important diagnosis to consider is neurogenic claudication. Usually patients have back pain or history of back pain. The pain may be reduced slightly by bending forward as with using a shopping cart. Pain is usually not relieved by just stopping walking as in patients with IC but requires patients to sit or lie down to relieve their pain. Venous Claudication is usually relieved by elevating the feet and is usually triggered by standing and sitting instead of walking. Musculoskeletal causes like arthritis, bursitis, tendinitis and myositis are common causes of lower extremity pain and could be differentiated from vascular claudication by detailed history and physical exam.

\section{Diagnosis}

Is usually made after taking detailed history, doing physical examination and noninvasive studies.

\section{Noninvasive indirect physiological tests}

Ankle-Brachial Index (ABI): Normally the pressures around the ankle are higher than the brachial pressures due to added aortic recoil pressure. The basic principal of $A B I$ is that the ratio of the higher ankle pressure over the higher of the brachial pressure should be one or above. To increase specificity of the test, 0.9 or 0.95 is used as a cut off for diagnosis of PAD. There are significant limitations on the value of $A B I$ related to non-compressible vessels secondary to medial calcifications, as in patients with diabetes and chronic kidney disease. Bedside $A B I$ check is quick, safe, and cheap and can be done without expensive equipment or significant time commitment and can be done by nursing staff with some training. It can be checked using simple a stethoscope and a blood pressure cuff. However hand held Doppler is more sensitive, especially in patients with PAD. A pressure difference between the brachial artery pressures of more than $12 \mathrm{mmHg}$ could be secondary to subclavian or innominate artery stenosis. Other causes are coarctation of aorta, vasculitis and external compression of the subclavian artery by tumor.

Toe Brachial Index (TBI): Is especially helpful in patients with arterial calcification where ABI can be falsely elevated or cannot be calculated. Digital arteries are not affected by arterial calcification. Normal toe brachial index range is 0.8-0.9. It could be abnormal in patients with digital arterial disease like in patients with Raynaud's phenomenon.

Segmental pressure measurement: Is done by measuring systolic pressure at the brachial, upper thigh, above knee, below knee and around the ankle. Vertical or horizontal pressure gradient of more than $20 \mathrm{mmHg}$ indicate a hemodynamically significant arterial stenosis. This is helpful in detecting PAD and sometimes the location of possible narrowing.

Segmental Pulse Volume Recordings (PVR): Normally demonstrate sharp systolic upstroke and decline during diastole. There is a retrograde flow during diastole that causes flow reversal and dicrotic notch. Loss of dicrotic notch is the earliest sign of arterial disease followed by delayed 
systolic upstroke and the peak is rounded and the down slope bowed away from baselines. It is especially useful in patients with arterial calcification and non-compressible vessels.

Digital Photoplethysmography (PPG): Tracings are important for diagnosing small vessels arterial disease like vasculitis and raynaud's. This is important especially in patients with normal $A B I$ but with signs and symptoms of digital ischemia.

Post-exercise ABIs: Are useful when resting ABIs are normal and the patient has symptoms of claudication or possible pseudoclaudication.

\section{Noninvasive direct studies that provides anatomic details}

Duplex ultrasonography (duplex ultrasound): Is used to diagnose PAD and to assess its severity and anatomical location. This includes real time B-mode (gray scale) imaging to best define vessels anatomy such as the presence of aneurysm and plaque as well as its morphology. Pulsed wave and color doppler imaging is used to detect blood flow, velocities and to estimate degree of stenosis.

CT or MR angiography: Are useful to assess severity and anatomy of PAD and choose appropriate endovascular or open revascularization.

\section{Invasive testing}

Arterial angiography: Is the gold standard for defining the anatomy of arterial disease. Sometimes patients undergo contrast angiography angiogram without CTA or MRA to minimize the amount of contrast dye received by the patients especially those with chronic kidney disease. Arterial angiography requires less contrast material than $\mathrm{CT}$ angiography. In addition, it provides an opportunity for endovascular intervention at the same setting.

\section{Case Report}

A 54-year-old man was seen for left calf pain that occurs after walking about two blocks on flat ground for at least six months. The pain is usually triggered at a shorter distance when walking at a faster pace or uphill and is relieved after resting for a few minutes. The pain extends to his left thigh if he continues to walk. He denied any pain in his feet or legs while resting or standing. He did not seek medical attention prior to this visit because he attributed the pain to being physically inactive. His

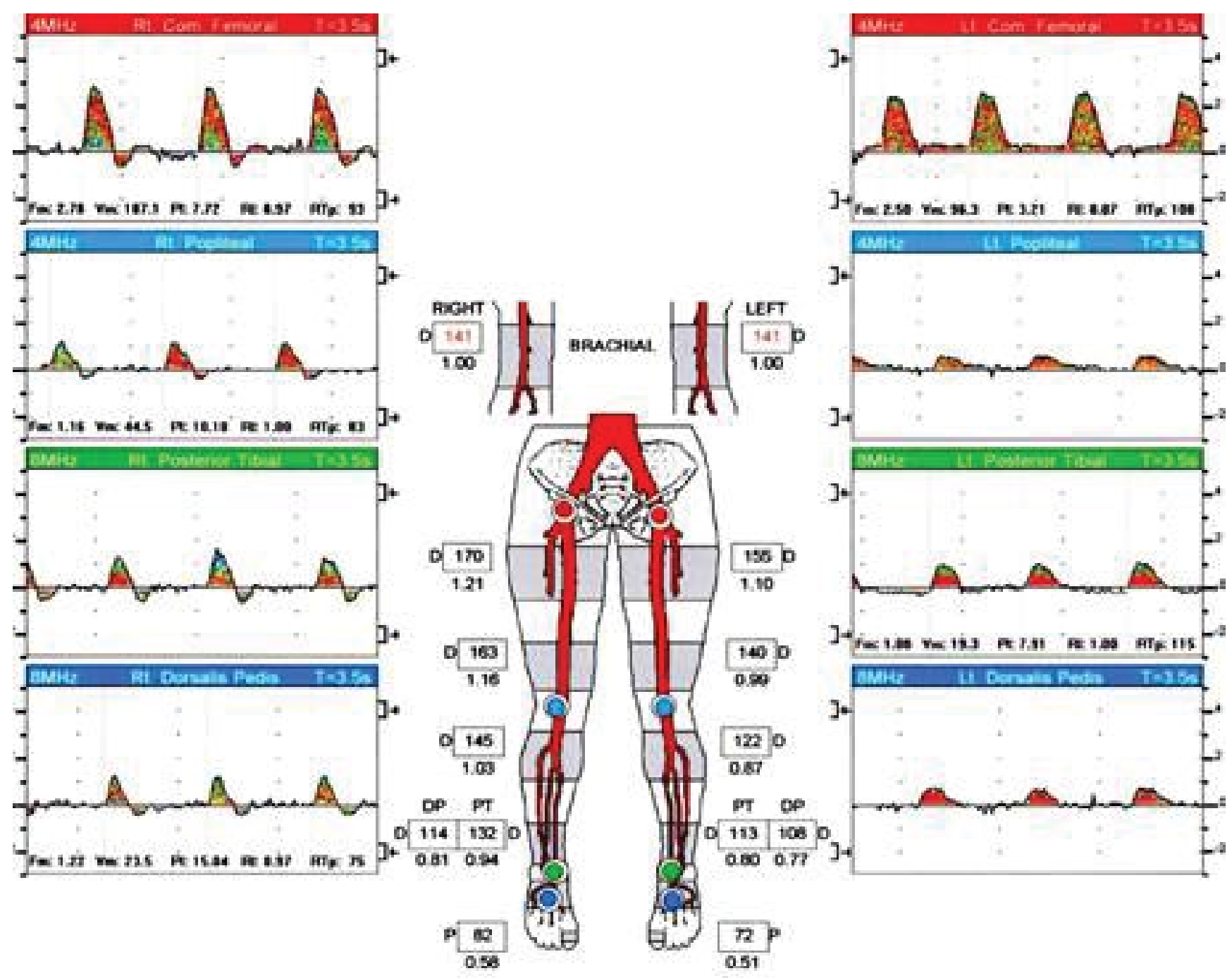

Figure 1: Lower extremity segmental study. 
past medical history includes hypertension, hyperlipidemia and tobacco use. He was on hydrochlorothiazide $25 \mathrm{mg} /$ day for hypertension. He had no allergies. His parents have hypertension and hyperlipidemia. He was single and lived alone. He did not graduate from high school and was working as a cashier in a supermarket. He has a 32 pack-year history of smoking. He does not drink. His review of symptoms was negative.

On examination, his blood pressure was 146/84 with pulse of 67 . His weight was 167 pounds. He has no neck vein distension or carotid bruit. Heart: normal S1 and S2, no murmurs, regular rate and rhythm. Chest: Clear to auscultation. The abdomen was soft without tenderness, guarding, mass, rebound or organomegaly. Bowel sounds were normal. No CVA tenderness or inguinal adenopathy. His feet were warm symmetrically, pink and well perfused with no clubbing, cyanosis, edema or signs of embolization or ulcers. On the right side his femoral, popliteal, dorsalis pedis and posterior tibial pulses were easily palpable and $2+$. On the left side his femoral, popliteal, dorsalis pedis and posterior tibial pulses were palpable but diminished and 1+. He had left femoral bruit. He had a normal range of motion of the knee and hip joints and there were no skin lesions. Lower extremity arterial segmental study was done and confirmed the presence of mild left lower extremity resting ischemia (Figure 1).

\section{Right leg}

The resting ankle-brachial index is 0.94 . The toe pressure is $82 \mathrm{mmHg}$ with a TBI of 0.58 Segmental pressures across the lower extremity demonstrate significant resting vertical pressure gradient. Doppler waveform analysis shows relatively normal arterial waveforms at all levels with preserved diastolic flow reversal. This represents borderline resting right lower extremity arterial study.

\section{Left leg}

The resting ankle-brachial index is 0.80 . The toe pressure is $72 \mathrm{mmHg}$ with a TBI of 0.51 . Segmental pressure at the high thigh level is normal. There is significant pressure gradient between the above knee popliteal pressure and the ankle level pressures. Doppler waveform analysis shows an abnormal monophasic high thigh waveform with continuous forward flow during the cardiac cycle. This represents mild left lower extremity arterial disease sec-

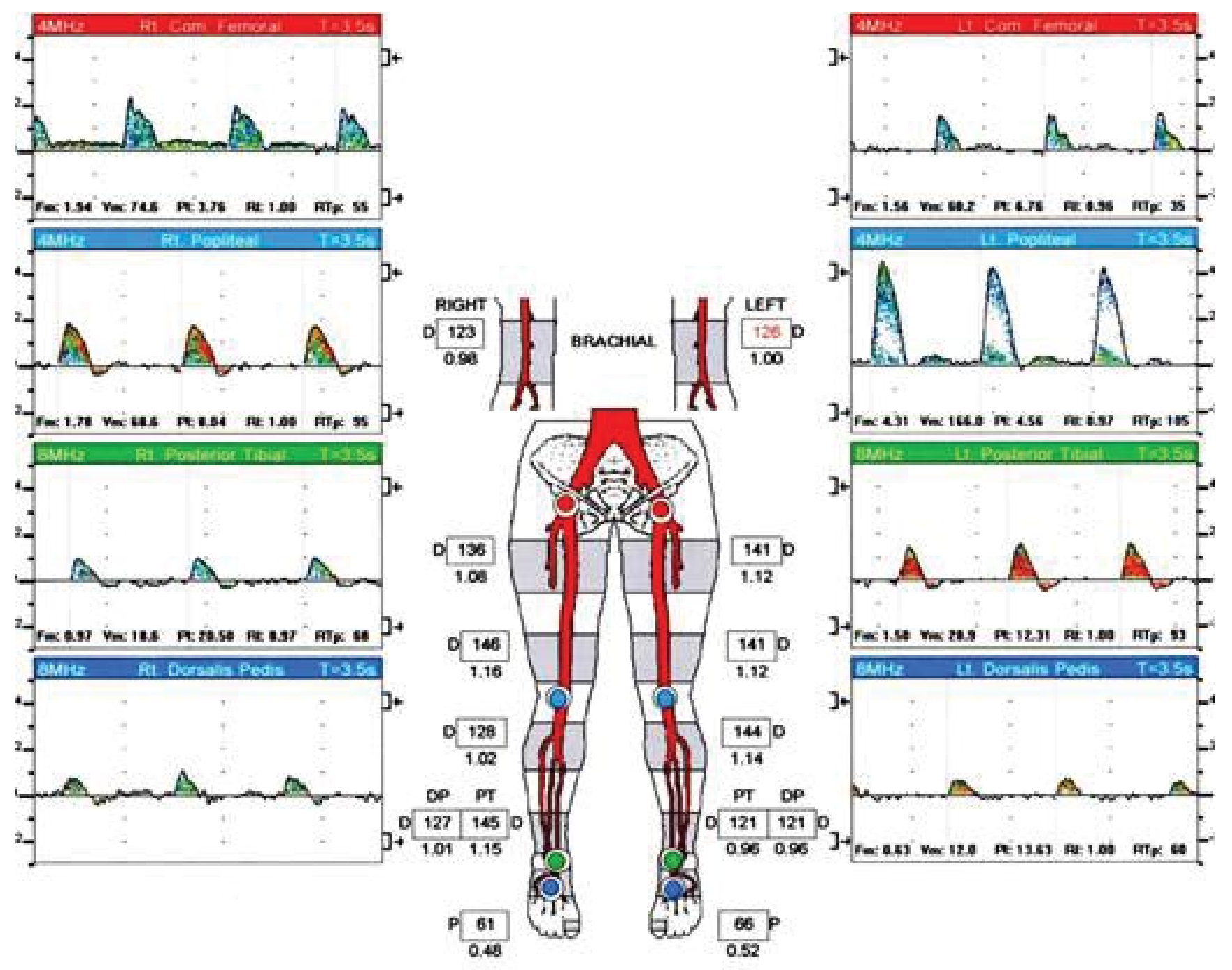

Figure 2: Lower extremity segmental study three years later. 
ondary to multilevel arterial disease (inflow disease and infrainguinal).

\section{Management}

In this case, the diagnosis of claudication secondary to atherosclerosis arterial disease with associated risks of limb loss, heart disease, stroke and death were discussed in detail with the patient. Therapeutic interventions were discussed in terms of targeting one of two main goals or both. The first goal is to reduce claudication symptoms and risk of future limb-related complications and second is to improve his health in general and to reduce his risk of heart disease, stroke, and death. Within that frame, indications, risks and potential benefits of every proposed intervention were discussed. It was emphasized to him that tobacco use is his number one risk factor and quitting smoking is the most important step he could take to prevent further progression of his peripheral arterial disease, reduce risks of heart disease, stroke and to improve his overall health. He was provided with smoking cessation counseling and was able to quit smoking three months after his first visit using nicotine patches and gum for four months. Blood tests were done as shown in Table 1. He was started on aspirin $81 \mathrm{mg}$, atorvastatin $40 \mathrm{mg}$ and lisinopril $20 \mathrm{mg}$ once daily. He was referred to dietary service to improve his blood pressure control and to lower his lipids.

He was advised to start doing regular exercises like walking and or biking. He was referred to physical therapy and although did not enroll in a physical rehabilitation program, he started to walk regularly. He was reassured that walking through his calf pain is not harmful to his health. Shortly after that he adopted a dog and started walking and later jogging with his dog on a regular daily basis. The time to onset of his left leg pain became progressively longer and the severity of the pain was milder. He was able to gradually increase his exercise level from two blocks at the time of his initial visit to jogging two miles almost every day three years later. Lower extremity segmental study three years later (Figure 2).

\section{Right leg}

The resting ankle-brachial index is 1.15 . The toe pressure is $61 \mathrm{mmHg}$ with a TBI of 0.48 . Segmental pressures across the lower extremity do not demonstrate any significant resting vertical or horizontal gradients. Doppler waveform analysis show relatively normal arterial waveforms at all levels with preserved diastolic flow reversal.

\section{Left leg}

The resting ankle-brachial index is 0.96 . The toe pressure is $66 \mathrm{mmHg}$ with a TBI of 0.52. Segmental pressures across the lower extremity demonstrate significant resting vertical and horizontal gradients. Doppler waveform analysis show relatively normal arterial waveforms at all levels with preserved diastolic flow reversal.

\section{Discussion}

Claudication is an important symptom of lower extremity arterial disease and more importantly is an indicator of systemic atherosclerosis with associated high cardiovascular events and death. Although limb related complications are frightening to patients, limb related prognosis is usually benign. Within five years, only $1-2 \%$ of patients (and likely less for those who adopt a healthy life-style) with claudication progress to develop more severe symptoms like rest pain, ischemic ulcers or gangrene and for whom revascularization is usually effective. However, $15-30 \%$ will die mostly secondary to cardiovascular disease and $25 \%$ will have a heart attack or a stroke $[5,6]$.

Also, patients with PAD have greater functional decline and mobility loss than those without PAD. Even patients with asymptomatic PAD were found to have poor functional capacities and poorer quality of life in comparison to those without PAD. Patients with claudication are more sedentary and their free-living daily physical activity was significantly lower than healthy subjects. Because of this functional decline, increased risks for cardiovascular events and ischemic complications like ulcers, gangrene and amputations and the associated need for hospitalizations, they have a poor quality of life and higher rates of depression than those without PAD [7-12].

So the main therapeutic goals in patients with claudication are to prevent cardiovascular events especially fatal and nonfatal $\mathrm{MI}$ and stroke, reduce limb-related symptoms and complications and improve functional abilities and quality of life. To help patients with claudication make an informed decision regarding their treatment and to motivate them to make necessary and difficult life style changes, the above goals need to be conveyed clearly to patients. Health care providers need to explain to their claudication patients that although their symptoms are related to their legs, the

Table 1: Three year Data Comparison (Blood pressure, cholesterol, estimated CV risk scores, $A B I$ and walking distance).

\begin{tabular}{|c|c|c|c|c|c|c|c|c|c|}
\hline & $\begin{array}{l}\text { Blood } \\
\text { pressure } \\
\mathrm{mmHg}\end{array}$ & $\begin{array}{l}\text { Total } \\
\text { cholesterol } \\
\text { mg/dl }\end{array}$ & $\begin{array}{l}\text { HDL } \\
\text { cholesterol } \\
\text { mg/dl }\end{array}$ & $\begin{array}{l}\text { TG } \\
\text { cholesterol } \\
\text { mg/dl }\end{array}$ & $\begin{array}{l}\text { LDL } \\
\text { cholesterol } \\
\text { mg/dl }\end{array}$ & $\begin{array}{l}\text { 10-year } \\
\text { framingham } \\
\text { CVD risk } \\
\text { score }\end{array}$ & $\begin{array}{l}\text { ACCIAHA } \\
201310 \text {-year } \\
\text { risk of heart } \\
\text { disease or } \\
\text { stroke }\end{array}$ & ABI & $\begin{array}{l}\text { Maximum } \\
\text { walking } \\
\text { distance }\end{array}$ \\
\hline $\begin{array}{l}\text { First } \\
\text { visit }\end{array}$ & $146 / 84$ & 281 & 35 & 169 & 212 & $>30 \%$ & $27.7 \%$ & $\begin{array}{l}\text { R: } 0.94 \\
L: 0.80\end{array}$ & $\begin{array}{l}\text { Two blocks on } \\
\text { a flat ground }\end{array}$ \\
\hline $\begin{array}{l}\text { Three } \\
\text { years } \\
\text { later }\end{array}$ & $125 / 69$ & 117 & 41 & 38 & 68 & $3 \%$ & $5.6 \%$ & $\begin{array}{l}\mathrm{R}: 1.15 \\
\mathrm{~L}: 0.96\end{array}$ & $\begin{array}{l}\text { Able to Run } 2 \\
\text { miles }\end{array}$ \\
\hline
\end{tabular}


most serious consequences of atherosclerosis are related to ischemic heart disease and stroke. In that sense, Paul Brand's statement that "Pain is God's greatest gift to mankind" applies to patients with claudication if viewed as an opportunity and a "teachable moment" to reduce cardiovascular events, improving overall health and reducing claudication symptoms. We need to help patients shift their focus from only their immediate presenting symptoms to the general risk on their health especially cardiovascular complication. This is not an easy task and requires multidisciplinary team and supportive health care systems placing significant emphasis on prevention.

The most important risk factors for PAD are tobacco use, obesity, diabetes, hypertension, hyperlipidemia and low kidney function $[13,14]$. Mortality rate was reduced by moderately vigorous exercise and quitting smoking by $23 \%$ and $41 \%$ respectively [15]. Risks of death, heart attack and amputations are significantly higher in patients with PAD who continue to smoke than those who are able to quit smoking [16-19]. Supervised treadmill exercise programs increased maximal treadmill walking time by $50-200 \%$ [20-23].

Furthermore, it was found that in adults who are at risk for development if Diabetes (BMI > 24 and fasting blood sugar $95-125 \mathrm{mg} / \mathrm{dl}$ or $145-199 \mathrm{mg} / \mathrm{dl}$ at the 2 hours after $75 \mathrm{gm}$ of oral glucose). 150 minutes of exercise every week and $7 \%$ weight reduction over 24 weeks resulted in 58\% reduction in incidence of DM in comparison to placebo $[24,25]$.

Statin [26-30] and ACEI [31,32] have been found to reduce mortality and possibly improve claudication symptoms. Available FDA approved medication to treat Claudication is cilostazol [33]. Pentoxifylline was not found to be effective in the treatment of claudication. Antiplatelet therapy has been a cornerstone of medical management of patients with symptomatic PAD as recommended by the latest AHA Guidelines. "Antiplatelet therapy with aspirin alone (range 75-325 mg per day) or clopidogrel alone (75 $\mathrm{mg}$ per day) is recommended to reduce $\mathrm{MI}$, stroke, and vascular death in patients with symptomatic PAD" [34]. Recent publications and advances in the use of newer antiplatelets and anticoagulants like Ticagrelor and Rivaroxaban in patients with PAD $[35,36]$ could potentially change future guidelines related to the management of patients with PAD. Revascularization (endovascular or open) is indicated in patients with significant lifestyle limiting claudication with inadequate response to optimal medical management. Medical management is crucial in these patients to reduce their risk of cardiovascular events. Revascularization has risks of preoperative mortality and morbidity and questionable long term patency [37,38]. Treatment selection should be individualized depending on patients' characteristic, arterial anatomy and risk of perioperative adverse events. Endovascular procedures should not be performed in patient with PAD to only prevent the progression to critical limb ischemia [36].

\section{Conclusion}

This case demonstrated clearly that optimal medical therapy resulted in the resolution of claudication symptoms but significant increase in his level of daily physical activities, risk reduction of cardiovascular diseases and significant improvement of his overall wellbeing. This is the most cost-effective treatment in patients with claudication. Revascularization is indicated for individuals with a lifestyle-limiting disability due to claudication if they do not have adequate response to medical management [5]. Although life-style changes are very challenging and not every patient is capable of achieving these goals, health care providers need to provide every patient with adequate information and support to allow them make informed decisions about their health and to motivate them to achieve their goals. Health care systems require providing essential support services like physical therapy, dietary and smoking cessation counseling. In the $21^{\text {st }}$ century, it is not enough to just tell the patients with claudication to "quit smoking and go walking" or to recommend revascularization without providing them with recommended optimal medical management.

\section{Conflict of Interest}

The author has no potential conflict of interest or financial relationship to disclose.

\section{References}

1. Murabito JM, Evans JC, Nieto K, Larson MG, Levy D, et al. (2002) Prevalence and clinical correlates of peripheral arterial disease in the Framingham Offspring Study. Am Heart J 143: 961-965.

2. Newman AB, Shemanski L, Manolio TA, Cushman M, Mittelmark M, et al. (1999) Ankle-arm index as a predictor of cardiovascular disease and mortality in the Cardiovascular Health Study. The Cardiovascular Health Study Group. Arterioscler Thromb Vasc Biol 19: 538-545.

3. Selvin E, Erlinger TP (2004) Prevalence of and risk factors for peripheral arterial disease in the United States: results from the National Health and Nutrition Examination Survey, 1999-2000. Circulation 110: 738-743.

4. Hirsch AT, Criqui MH, Treat-Jacobson D, Regensteiner JG, Creager MA, et al. (2001) Peripheral arterial disease detection, awareness, and treatment in primary care. JAMA 286: 1317-1324.

5. Dormandy J, Heeck L, Vig S (1999) The natural history of claudication: risk to life and limb. Semin Vasc Surg 12: 123-137.

6. Hirsch AT, Haskal ZJ, Hertzer NR, Bakal CW, Creager MA, et al. (2006) ACC/AHA 2005 Practice Guidelines for the management of patients with peripheral arterial disease (lower extremity, renal, mesenteric, and abdominal aortic): a collaborative report from the American Association for Vascular Surgery/Society for Vascular Surgery, Society for Cardiovascular Angiography and Interventions, Society for Vascular Medicine and Biology, Society of Interventional Radiology, and the ACC/AHA Task Force on Practice Guidelines (Writing Committee to Develop Guidelines for 
the Management of Patients With Peripheral Arterial Disease): endorsed by the American Association of Cardiovascular and Pulmonary Rehabilitation; National Heart, Lung, and Blood Institute; Society for Vascular Nursing; TransAtlantic Inter-Society Consensus; and Vascular Disease Foundation. Circulation 113: e463-e654.

7. McDermott MM, Greenland P, Guralnik JM, Liu K, Criqui $\mathrm{MH}$, et al. (2003) Depressive symptoms and lower extremity functioning in men and women with peripheral arterial disease. J Gen Intern Med 18: 461-467.

8. McDermott MM, Guralnik JM, Ferrucci L, Tian L, Liu K, et al. (2008) Asymptomatic peripheral arterial disease is associated with more adverse lower extremity characteristics than intermittent claudication. Circulation 117: 2484-2491.

9. McDermott MM, Jack M Guralnik, Lu Tian, Kiang Liu, Luigi Ferrucci, et al. (2009) Associations of borderline and low normal ankle-brachial index values with functional decline at 5-year follow-up: the WALCS (Walking and Leg Circulation Study). J Am Coll Cardiol 53: 1056-1062.

10. McDermott MM, Liu K, Greenland P, Guralnik JM, Criqui $\mathrm{MH}$, et al. (2004) Functional decline in peripheral arterial disease: associations with the ankle brachial index and leg symptoms. JAMA 292: 453-461.

11. Regensteiner JG, Hiatt WR, Coll JR, Criqui MH, Treat-Jacobson D, et al. (2008) The impact of peripheral arterial disease on health-related quality of life in the Peripheral Arterial Disease Awareness, Risk, and Treatment: New Resources for Survival (PARTNERS) Program. Vasc Med 13: 15-24.

12. Sieminski DJ, Gardner AW (1997) The relationship between free-living daily physical activity and the severity of peripheral arterial occlusive disease. Vasc Med 2: 286-291.

13. MacGregor AS, Price JF, Hau CM, Lee AJ, Carson MN, et al. (1999) Role of systolic blood pressure and plasma triglycerides in diabetic peripheral arterial disease. The Edinburgh Artery Study. Diabetes Care 22: 453-458.

14. Selvin E, Hirsch AT (2008) Contemporary risk factor control and walking dysfunction in individuals with peripheral arterial disease: NHANES 1999-2004. Atherosclerosis 201: 425-433.

15. Paffenbarger RS, Hyde RT, Wing AL, Lee IM, Jung DL, et al. (1993) The association of changes in physical-activity level and other lifestyle characteristics with mortality among men. N Engl J Med 328: 538-545.

16. Faulkner KW, House AK, Castleden WM (1983) The effect of cessation of smoking on the accumulative survival rates of patients with symptomatic peripheral vascular disease. Med J Aust 1: 217-219.

17. Jonason T, Bergstrom R (1987) Cessation of smoking in patients with intermittent claudication. Effects on the risk of peripheral vascular complications, myocardial infarction and mortality. Acta Med Scand 221: 253-260.

18. Lassila R, Lepantalo M (1988) Cigarette smoking and the outcome after lower limb arterial surgery. Acta Chir Scand 154: 635-640.

19. Quick CR, Cotton LT (1982) The measured effect of stopping smoking on intermittent claudication. Br J Surg 69: S24-S26.

20. Regensteiner JG (1997) Exercise in the treatment of claudication: Assessment and treatment of functional impairment. Vasc Med 2: 238-242.

21. Leng GC, Fowler B, Ernst E (2000) Exercise for intermittent claudication. Cochrane Database Syst Rev.

22. Hall JA, James BJ (1982) The effects of an intensive 26day program of diet and exercise on patients with peripheral vascular disease. J Cardiac Rehabil 2: 569-574.

23. Gardner AW, Poehlman ET (1995) Exercise rehabilitation programs for the treatment of claudication pain. A me- ta-analysis. JAMA 274: 975-980.

24. Diabetes Prevention Program Research Group (2002) Reduction in the incidence of type 2 diabetes with lifestyle intervention or metformin. N Engl J Med 346: 393-403.

25. Watson L, Ellis B, Leng GC (2008) Exercise for intermittent claudication. Cochrane Database Syst Rev.

26. Pedersen TR, Kjekshus J, Pyörälä K, Olsson AG, Cook TJ, et al. (1998) Effect of simvastatin on ischemic signs and symptoms in the Scandinavian simvastatin survival study (4S). Am J Cardiol 81: 333-335.

27. Mondillo S, Ballo P, Barbati R, Guerrini F, Ammaturo T, et al. (2003) Effects of simvastatin on walking performance and symptoms of intermittent claudication in hypercholesterolemic patients with peripheral vascular disease. Am J Med 114: 359-364.

28. Mohler ER 3rd, Hiatt WR, Creager MA (2003) Cholesterol reduction with atorvastatin improves walking distance in patients with peripheral arterial disease. Circulation 108: 1481-1486.

29. Buchwald H, Bourdages HR, Campos CT, Nguyen P, Williams SE, et al. (1996) Impact of cholesterol reduction on peripheral arterial disease in the Program on the Surgical Control of the Hyperlipidemias (POSCH). Surgery 120: 672-679.

30. Aronow WS, Nayak D, Woodworth S, Ahn C (2003) Effect of simvastatin versus placebo on treadmill exercise time until the onset of intermittent claudication in older patients with peripheral arterial disease at six months and at one year after treatment. Am J Cardiol 92: 711-712.

31. Heart Outcomes Prevention Evaluation Study Investigators, Yusuf S, Sleight P, Pogue J, Bosch J, et al. (2000) Effects of an angiotensin-converting-enzyme inhibitor, ramipril, on cardiovascular events in high-risk patients. The Heart Outcomes Prevention Evaluation Study Investigators. N Engl J Med 342: 145-153.

32. Ahimastos AA, Walker PJ, Askew C (2013) Effect of ramipril on walking times and quality of life among patients with peripheral artery disease and intermittent claudication: A randomized controlled trial. JAMA 309: 453-460.

33. Stevens JW, Simpson E, Harnan S, Squires H, Meng Y, et al. (2012) Systematic review of the efficacy of cilostazol, naftidrofuryl oxalate and pentoxifylline for the treatment of intermittent claudication. Br J Surg 99: 1630-1638.

34. Gerhard-Herman, Heather L Gornik, Coletta Barrett, Neal R Barshes, Matthew A Corriere, et al. (2016) 2016 AHA/ACC Guidelines on the Management of Patients with Lower Extremity Artery Disease: Executive Summary. Circulation 136: 11.

35. Hiatt WR, Fowkes FG, Heizer G, Berger JS, Baumgartner I, et al. (2017) Ticagrelor versus clopidogrel in symptomatic peripheral artery disease. N Engl J Med 376: 32-40.

36. John W Eikelboom, Stuart J Connolly, Jackie Bosch, Gilles R Dagenais, Robert G Hart, et al. (2017) Rivaroxaban with or without aspirin in stable cardiovascular disease. N Engl J Med.

37. Amritha Raghunathan, Joseph H Rapp, Fred Littooy, Steven Santilli, William C Krupski, et al. (2006) Postoperative outcomes for patients undergoing elective revascularization for critical limb ischemia and intermittent claudication: a subanalysis of the Coronary Artery Revascularization Prophylaxis (CARP) trial. J Vasc Surg 43: 1175-1182.

38. Nordanstig J, Gelin J, Hensäter M, Taft C, Österberg K, et al. (2011) Walking performance and health-related quality of life after surgical or endovascular invasive versus non-invasive treatment for intermittent claudication--a prospective randomised trial. Eur J Vasc Endovasc Surg 42: 220-227. 